\title{
Experimental Investigation of VSS in Virtual Red Blood Cells and Platelets in the Flow of a PVAD Using Pseudo-Tracking Analysis
}

\author{
Bernardo Luiz Harry Diniz Lemos', Vítor Augusto Andreghetto Bortolin¹, Rodrigo de Lima Amaral ${ }^{1}$, \\ Marcelo Mazzeto ${ }^{2}$, Idágene Aparecida Cestari ${ }^{2}$, Júlio Romano Maneghini ${ }^{1}$ \\ ${ }^{1}$ Escola Politécnica da Universidade de São Paulo (EP USP) \\ Av. Prof. Mello Moraes 2231, São Paulo, Brazil \\ bernardolemos@usp.br; vitor.bortolin@usp.br; rodrigoamaral@usp.br; jmeneg@usp.br \\ ${ }^{2}$ Bioengineering Laboratory, Heart Institute, Hospital das Clínicas, HCFMUSP, Faculdade de Medicina, Universidade de \\ São Paulo \\ Av. Dr. Enéas Carvalho de Aguiar 44, São Paulo, Brazil \\ simao.bacht@incor.usp.br; marcelo.mazzetto@incor.usp.br; idagene.cestari@incor.usp.br
}

\begin{abstract}
Ventricular assist devices (VAD) improve patient's survival rates at the heart transplant waiting list. Notwithstanding, VADs are not perfect replacements for a failing heart having their design and operational issues resulting in hemolysis and thrombogenesis. Both blood degradation processes are associated with viscous shear stress (VSS). Therefore, one way to experimental evaluate VSS values along residence time that red blood cells and platelets are subject to is pseudo-tracking. In pseudo-tracking method a postprocessing is done in particle image velocimetry (PIV) data, so particles can be generated inside the velocity field and their pathlines will provide VSS values. For better accuracy in this Lagrangian approach, temporal resolution is a must. Therefore, time-resolved PIV (TRPIV) technique for the VAD is a solution. In this work, the pneumatic pediatric assist device (PVAD) developed by Instituto do Coração (InCor) was measured using a TR-PIV at 70 beats per minute (bpm), and a pseudo-tracking analysis developed at MATLAB was done with two reconstructed particles. The first reconstructed particle was created near the inlet valve and the second near the outlet valve. Both reconstructed particles were generated at the beginning of PVAD's diastole and their instantaneous and integrated VSS with resident time values were compared to literature thresholds for erythrocytes and thrombocytes.
\end{abstract}

Keywords: Ventricular Assist Device. Viscous Shear Stress. Erythrocytes. Thrombocytes. Pseudo-Tracking.

\section{Introduction}

Some of the patients with cardiovascular diseases progress to heart failure and, in the most severe cases, end up needing a heart transplant. However, the number of organs available for transplants is less than necessary. The ventricular assist device (VAD) guarantees a longer survival to the patients [1]-[3]. However, VADs have problems related to their design and operating conditions resulting in hemolysis and thrombogenesis. Blood degradation can be associated with the physical phenomena in internal flow, such as viscous shear stresses (VSS) in certain regions of these devices. In order to quantify the stresses blood is subject when cardiovascular devices are introduced in human body, in-vitro test with animal blood and model fluid have been conducted since the late 1960s. Experiments varying from a Couette [4] or cone-and-plate viscometer [5] to mock circulation loops (MCL) with hot-film anemometry (HFA) [6] and laser-Doppler anemometry (LDA) [7] measure, indirectly, the VSS and, directly, the resident time of blood in these setup. More recent, computational fluid dynamics (CFD) [8] was used to simulate shear stresses and resident time in continuous flow VADs. Although the numerous efforts in lysis experiments, there is no consensus in threshold values for VSS or resident time for erythrocytes and thrombocytes. It is also important to emphasize that, apart from CFD, the experiments were not capable to trace the displacement of single cells, as they were done by sampling.

One experimental possibility to assess this concern is within time resolved particle image velocimetry (TR-PIV). After measurements of cardiovascular devices, for example VADs and valves, PIV is capable to depict an instantaneous twodimensional velocity field and, with post-processing all other types of velocity-based properties within the flow. Now, with the temporal resolution propitiated by TR-PIV, a virtual particle generated in the flow representing a haematid or a platelet can be calculated with required accuracy. This method is called pseudo-tracking and is based on Lagrangian approach [9, 
10]. Notice that reconstructed particle paths are not available at the time of measurement, but are calculated at a later stage. With the pathline the instantaneous viscous shear stress (VSS) at each position can be assigned to the reconstructed particle and after compared with literature thresholds. In this situation, the pseudo-tracking analysis is an extension to PIV analysis with low computational cost and, similarly to CFD, can show regions where VSS are high.

In this work, the pneumatic pediatric assist device (PVAD) developed by InCor [11] was measured using a TR-PIV at 70 beats per minute (bpm), and after the last post-processing, a pseudo-tracking analysis elaborated at MATLAB was done with two reconstructed particles. The first reconstructed particle was created near the inlet valve and the second near the outlet valve. Both created particles were generated at the onset of PVAD's diastole.

\section{Experimental Methodology}

\subsection{PVAD and $M C L$}

The PVAD (Fig. 1a) was developed by Bioengineering Division research group at Instituto do Coração do Hospital das Clínicas da Faculdade de Medicina da Universidade de São Paulo (InCor). The PVAD model studied has a flexible diaphragm, dividing the blood and pneumatic chamber, in addition to bileaflet mechanical heart valves in both in- and outflows. The PVAD was adapted with a wedge shaped (Fig. 1b) outside the blood chamber for the experiments. The straight faces in the wedge shape ensure the perpendicular angle between the laser sheet and the field of view inside PVAD. Inside the device, the geometry was kept untouched, as well as the pneumatic chamber, so the ejection volume remained $30 \mathrm{~cm}^{3}$. For optical reasons the wedged PVAD was made of transparent polyurethane.

The hydropneumatics system, also known as mock circulation loop (MCL), is responsible to mimic the human hydrodynamic parameters. For the experiments, the MCL was connected in PVAD by two cannulas as illustrated in Fig. $1 \mathrm{~b}$. The inlet cannula comes from the atrial compliance chamber, which simulates the left atrium with a preload pressure of $5 \mathrm{mmHg}$. The outlet cannula was connected to an arterial chamber, responsible to maintain an afterload pressure of $120 \mathrm{mmHg}$ for systole and $80 \mathrm{mmHg}$ for diastole. Amid the atrial and arterial chambers were a systemic volume chamber open to atmosphere and a Hoffman clamp to adjust the systemic resistance to blood flow. A pulse generator coupled to a pneumatic actuator, maintaining $70 \mathrm{bpm}$, controlled the PVAD's inflation and deflation. Other parameters were set in the MCL, such as systolic duration of $260 \mathrm{~ms}$ (30\% of a whole beat), end diastolic delay (EDD) of $44 \mathrm{~ms}$ and end systolic delay of $14 \mathrm{~ms}$.
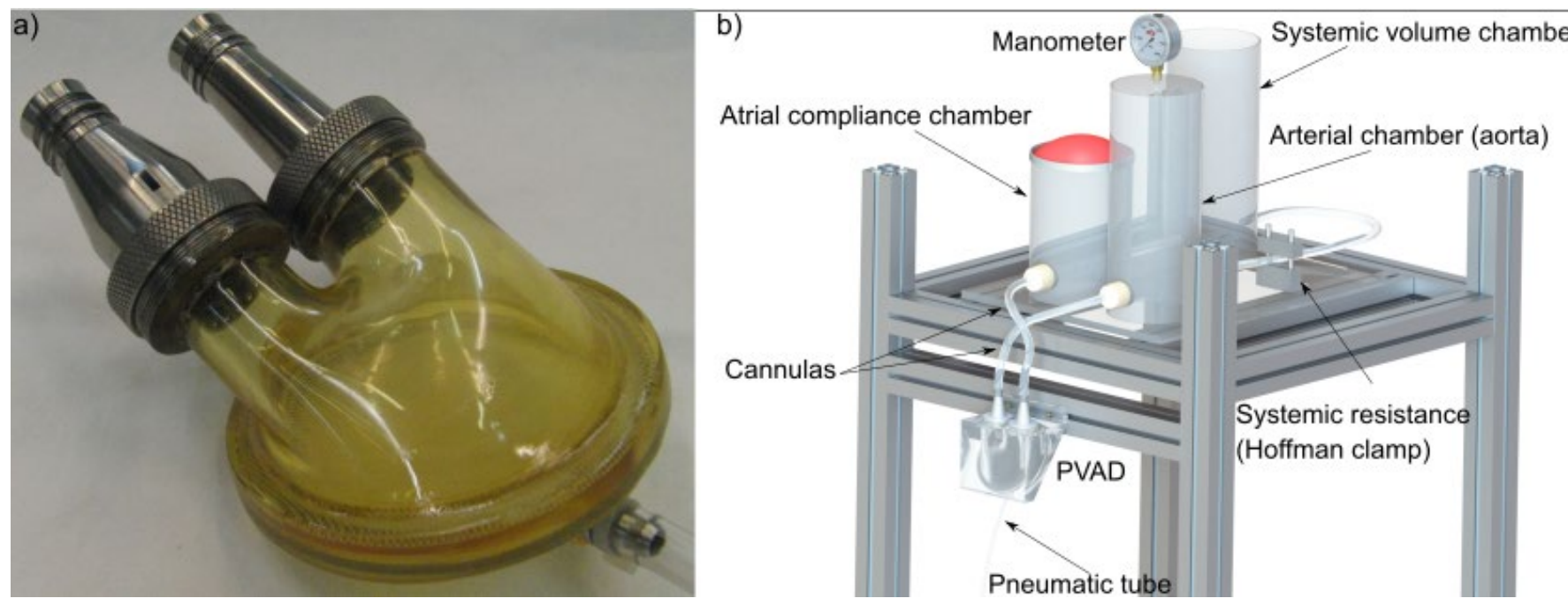

Fig. 1: InCor's PVAD and experimental setup 


\subsection{Data acquisition}

The PIV system is composed by the flow set with seeding particles, lighting, recording, calibration and processing. The fluid used to mimic blood was a mixture of $60 \%$ saline and $40 \%$ glycerol with $30 \mu \mathrm{m}$ in diameter polyamide particles. Despite the refractive index of 1.41, this liquid has properties similar to blood such as dynamic viscosity of $3.3 \times 10-3 \mathrm{~Pa}$.s and density of $1100 \mathrm{~kg} / \mathrm{m}$.

The laser system chosen for the time-resolved PIV (TR-PIV) consisted of a diode-pumped solid-state (DPSS) mediated by neodymium-doped yttrium lithium fluoride (Nd:YLF) with double cavity (Litron Lasers Ltd, LDY304). Other laser beam characteristics were: wavelength of $527 \mathrm{~nm}$ with band diameter of $5 \mathrm{~mm}$ and divergence less than $3 \mathrm{mrad}$. In addition, the laser frequency was set to $3250 \mathrm{~Hz}$ producing an energy of approximately $12.6 \mathrm{~mJ}$.

All Images were recorded in single-frame mode utilizing a complementary metal-oxide-semiconductor (CMOS) camera (Vision Research, Phantom ${ }^{\circledR}$ Miro ${ }^{\circledR}$ R311) with a sensor size of 1280 x 800 px. A set of lens and filters were assembled to prevent noise. A $527 \mathrm{~nm}$ with full width at half maximum (FWHM) of $5 \mathrm{~nm}$ bandpass filter and with optical density (OD) with a minimum value of 4 and minimum transmittance greater than $85 \%$ (Edmund Optics, 2017) was mounted to the camera. To synchronize all systems, a digital delay/pulse generator (Berkeley Nucleonics Corp (BNC), Model 575) was necessary during acquisition.

\subsection{Data Processing and Pseudo-Tracking Analysis}

The raw recorded images were calibrated to avoid distortions caused by the complex internal geometry of the PVAD and later a series of pre-processes filters were applied to remove noise. Henceforth, the final pass of standard cross correlation (SCC) was done in an interrogation window whose size was $15 \mathrm{px}$ with $25 \%$ of window overlap, producing vectors with 11 px after SCC. Post-processing was performed in order to remove outliers bigger than three times the median absolute deviation (MAD) in a 3 x 3 px interrogation window cluster.

The Lagrangian approach becomes especially valid for this work due to the temporal resolution obtained by TR-PIV [9]. Therefore, a pseudo-tracking algorithm was used as an approximation of the trajectory performed by the blood cells in the plane. Although is important to highlight the awareness of the three-dimensional movement of the flow in the device will make portions of the fluid to leave the observed plain. To calculate the pathlines along the cycle, first an initial location inside the velocity field is choose to be the origin of the massless particle. Then at each time step the direction and magnitude of the velocity in that specific point is calculated by interpolating the PIV field. To move the fictitious particle between time steps a Euler integration like approach was used, with the displacement being calculated as the initial velocity times the time step. Then, in the next point the process is repeated with the velocity of that particular region resulting in a complete particle trajectory (eq. 1) until the particle leaves the observed region.

$$
\overrightarrow{x_{p}}=\int \overrightarrow{u_{p}}\left(\overrightarrow{x_{p}}, t\right) d t \approx \overrightarrow{x_{(p, n+1)}}=\sum_{1}^{n} \overrightarrow{u_{p}}\left(\overrightarrow{x_{(p, n)}}, t_{n}\right) * \Delta t
$$

Being $x_{p}$ the position and $u_{p}$ the velocity of the fictitious particle, and $t$ is the time. The $n$ is the first time step and $n+1$ is the next time step.

Alongside the instantaneous temporal evolution, the effect of the stresses on this reconstructed particle must be accounted during its passage through the device. That is, the instantaneous stress at each point is storage with the position. Therefore, a curve is created with stress over time for that particular trajectory, then integrating the curve it is calculated the total shear suffer by that portion of the fluid through the device. In this context, the sum found was compared with the threshold values defined by the literature for hemolysis [4]-[8].

First, in this study, the geometric place and the initial instant in which a particle would be created and released in the flow is selected. This is a manual process and the main decision criteria was to avoid regions with high noise, like the shadow of the membrane, to ensure the quality of the data. After the creation of this reconstructed particle, its trajectory was calculated using the described algorithm implemented in MATLAB determined by the velocity vector provided by the PIV's 
instantaneous and two-dimensional velocity field. The pathline ends when the reconstructed particle leaves the device or enters a spurious data region corresponding to diaphragm shadow and PVAD's valves and walls. The instantaneous viscous shear stress (VSS) for two-component and two-dimensional PIV [7, 8] is calculated by equation 2.

$$
V S S=\mu\left(\frac{\partial u}{\partial y}+\frac{\partial v}{\partial x}\right)
$$

Where $\mu$ is the dynamic viscosity of the fluid, $\partial \mathrm{u} / \partial \mathrm{y}$ is the shear rate considering $\mathrm{x}-\mathrm{axis}$ and $\partial \mathrm{v} / \partial \mathrm{x}$ is the shear rate for y-axis.

The particle generation and area calculus were programmed in MATLAB after the post-processing. In order to perform this analysis, two initial points were selected based on PVAD's internal geometry and were generated at the beginning of diastole $(0 \%$ of a whole cardiac cycle).

\section{Results}

Two particles were created, the first one namely A and the second, B. Particle A represents the evolution of the inlet jet, since during all the time it remains very close to the external entrance wall (black line) for almost the entire initial half of the diastole (Fig. 2a). The reconstructed particle covered a distance of approximately $35 \mathrm{~mm}$ in $46 \%$ of the diastole (Fig. 2b) in its pathline. Higher VSS values were expected due to the proximity to the wall and the flow generated by the diastole, however the particle was not close enough to the wall to reach such magnitudes, resulting in a maximum VSS of $0.06 \mathrm{~Pa}$ and an area of 6.5 Pa.ms (Fig. 2c).

Otherwise, particle B represents the regurgitation in the output valve during diastole (Fig. 2d). Shortly after the $40 \%$ diastole mark, the reconstructed particle left the device, covering almost $12 \mathrm{~mm}$ (Fig. 2e) in its pathline. The VSS (Fig. 2f) of the B route are smaller than those found at point A, reaching $0.035 \mathrm{~Pa}$ and an area of 2.61 Pa.s.

The VSS values found in point A and B suggest that the flow inside the PVAD is probably not hemolytic. The pseudo-tracking approach exhibited values below the thresholds found in the literature for red blood cells and platelets. 

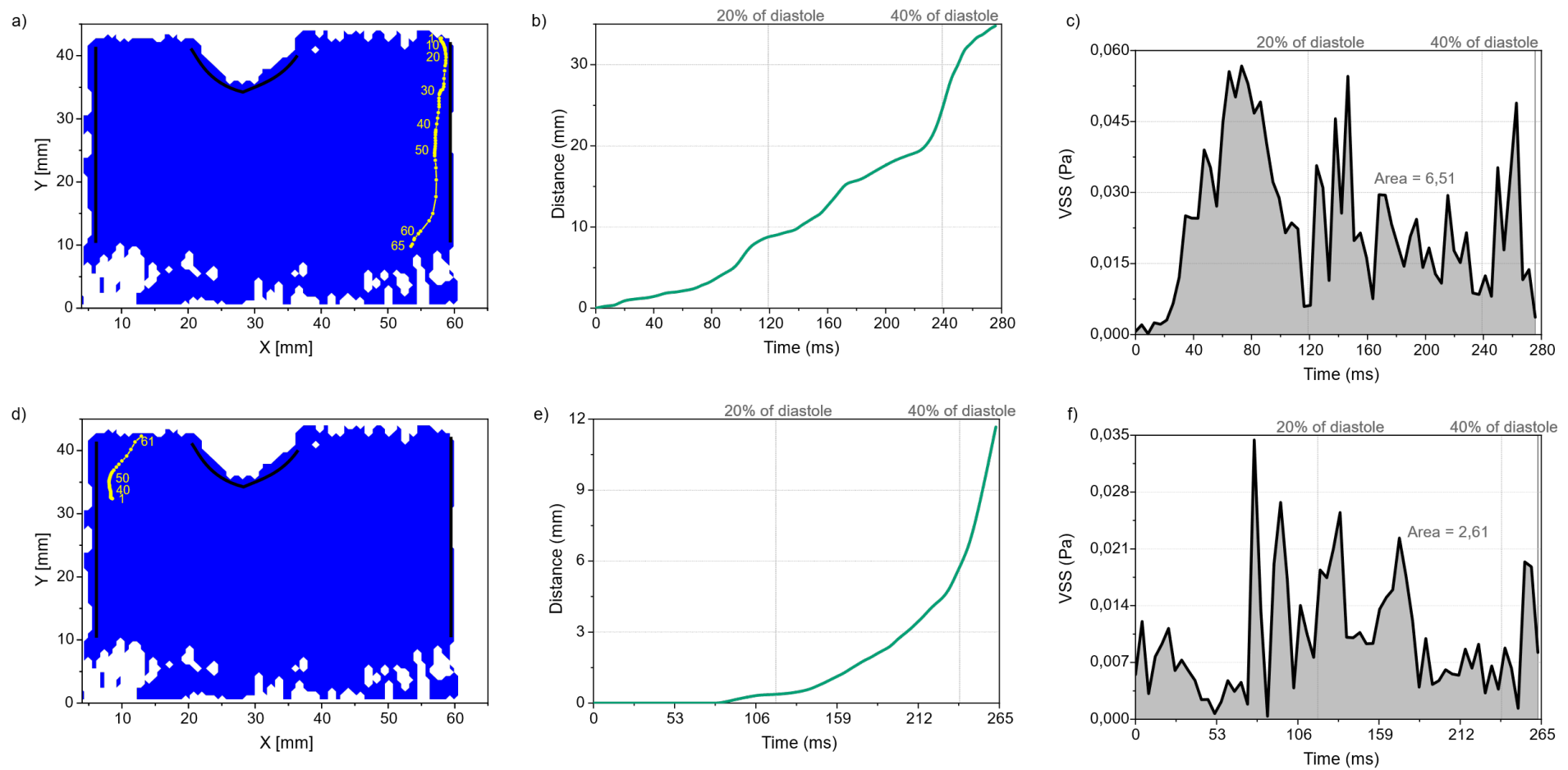

Fig. 2: Temporal evolution of reconstructed particle A and B

\section{Conclusion}

The pseudo-tracking approach was used for instant and cumulative VSS generated particles. Pseudo-tracking is a mathematical approach considered valid because the TR-PIV was a high temporal resolution. The literature stipulates maximum stress values and exposure times that cells are subjected to before disruption, during viscous shear stress (VSS). Platelets are more sensitive to shear stresses than red cells, with a limit for lysis in the order of $10 \mathrm{~Pa}$. The data indicates that the flow inside the PVAD is not hemolytic, due to the low values of instantaneous and cumulative VSS in temporal evolution.

\section{Acknowledgements}

We gratefully acknowledge support of the RCGI - Research Centre for Gas Innovation, hosted by the University of São Paulo (USP) and sponsored by FAPESP - São Paulo Research Foundation (2014/50279-4) and Shell Brasil, and the strategic importance of the support given by ANP (Brazil's National Oil, Natural Gas and Biofuels Agency) through the R\&D levy regulation. This study was financed in part by the Coordenação de Aperfeiçoamento de Pessoal de Nível Superior - Brasil (CAPES) - Finance Code 001. This work was carried out with support from the Conselho Nacional de Desenvolvimento Científico e Tecnológico (CNPq), process 311191/2017-7. This work was supported by grant \#2012/50283-6, São Paulo Research Foundation (FAPESP). We thank the support of Financiadora de Estudos e Projetos (FINEP).

\section{References}

[1] D. G. Tang, P. E. Oyer and H. R. Mallidi, "Ventricular Assist Devices: History, Patient Selection, and Timing of Therapy," Journal of Cardiovascular Translational Research, vol. 2, pp. 159-167, 19032009.

[2] D. G. Jakovljevic, M. H. Yacoub, S. Schueler, G. A. MacGowan, L. Velicki, P. M. Seferovic, S. Hothi, B.-H. Tzeng, D. A. Brodie, E. Birks and L.-B. Tan, "Left Ventricular Assist Device as a Bridge to Recovery for Patients With Advanced Heart Failure," Journal of The American College of Cardiology, vol. 69, no. 15, pp. 1924-1933, 2017. 
[3] S. Raju, J. MacIver, F. Foroutan, C. Alba, F. Billia and V. Rao, "Long-term use of left ventricular assist devices: a report on clinical outcomes," Canadian Journal of Surgery, vol. 60, no. 4, pp. 236-246, 2017.

[4] G. Heuser and R. Opitz, "A Couette Viscometer for Short Time Shearing of Blood," Biorheology, vol. 17, pp. 17-24, 1980.

[5] J. D. Hellums, “1993 Whitaker Lecture: Biorheology in Thrombosis Research,” Annals of Biomedical Engineering, vol. 22, pp. 445-455, 1994.

[6] W. Tillmann, H. Reul, M. Herold, K. -H. Bruss and J. v. Gilse, "In-vitro wall shear measurements at aortic valve prostheses," Journal of Biomechanics, vol. 17, no. 4, pp. 263-279, 1984.

[7] M. Giersiepen, L. J. Wurzinger, R. Opitz and H. Reul, "Estimation of shear stress-related blood damage in heart valve prostheses - in vitro comparison of 25 aortic valves," The International Journal of Artificial Organs, vol. 13, no. 5, pp. 300-306, 1990.

[8] K. H. Fraser, T. Zhang, M. E. Taskin, B. P. Griffith and Z. J. Wu, "A Quantitative Comparison of Mechanical Blood Damage Parameters in Rotary Ventricular Assist Devices: Shear Stress, Exposure Time and Hemolysis Index," Journal of Biomechanical Engineering, vol. 134, pp. 081002-1 - 081002-11, 082012.

[9] P. L. van Gent, F. F. J. Schrijer and B. W. van Oudheusden, "Assessment of the pseudo-tracking approach for the calculation of material acceleration and pressure fields from time-resolved PIV: part I. Error propagation," Measurement Science and Technology, vol. 045204, pp. 1-14, 732018.

[10] P. L. van Gent, F. F. J. Schrijer and B. W. van Oudheusden, "Assessment of the pseudo-tracking approach for the calculation of material acceleration and pressure fields from time-resolved PIV: part II. Spatio-temporal filtering," Measurement Science and Technology, vol. v. 29, no. 4, p. p. 045206, 932018.

[11] V. A. A. Bortolin, B. L. H. D. Lemos, R. d. L. Amaral, S. Bacht, M. Mazzeto, I. A. Cestari and J. R. Maneghini, "Temporal Flow Evolution on a Pediatric Ventricular Assist," in 7th International Conference of Fluid Flow, Heat and Mass Transfer, Niagara Falls, 2020. 